\title{
Viszeralchirurgie - quo vadis ChIRURGische GASTROENTEROLOGIE INTERDISZIPLINÄR?*
}

\author{
Reinhard Bittner \\ Klinik für Allgemein- und Visceralchirurgie, Marienhospital Stuttgart, Deutschland
}

Wir befinden uns in einer Zeit des Umbruchs!

Betriebswirtschaftliche Kriterien drohen die Fachkompetenz zu ersticken. Ein leitender Chirurg muss sich zu 70-80\% mit Managementaufgaben und nur zu 20-30\% mit klinisch-fachlichen Aufgaben befassen. Hinzu kommen schlechte Arbeitsbedingungen, eine katastrophal niedrige Vergütung sowie mangelhafte Perspektiven für den chirurgischen Nachwuchs mit den daraus resultierenden Folgen. Immer wieder und überall wird ein trostloses Bild von der deutschen Chirurgie gezeichnet. Statt in Ausbildung und Weiterentwicklung zu investieren, fließen die Gelder in die verschiedensten Verwaltungsbürokratien, zu Unternehmensberatern und «Zertifizierungshelfern». Schwierigkeiten bei der Gewinnung des chirurgischen Nachwuchses müssen zwangsläufige Folgen dieser Entwicklung sein.

Ist die Lage wirklich so katastrophal und hoffnungslos? Kein Zweifel - wir sind mit einem ganzen Strauß von Problemen konfrontiert. Aber sind diese wirklich unlösbar und berechtigen sie zu einer derart depressiven und mutlosen Stimmungslage?

Ich spreche immer wieder mit jungen Chefärzten. Durchweg vermitteln diese Gespräche erstaunlicherweise eine zufriedene Stimmung. Dies entspricht auch dem Ergebnis einer vom Konvent Leitender Krankenhauschirurgen initiierten, großen Umfrage. Die jüngeren Kollegen sind deutlich zufriedener als die älteren. Und, besonders bemerkenswert, die Chefärzte in den neuen Bundesländern sind zufriedener als die in den alten. Für das Wohlbefinden spielen offenbar neben den objektiven Gegebenheiten auch Gewohnheiten, Erwartungshaltung und Flexibilität eine Rolle.

\footnotetext{
* Mit Auszügen aus der Eröffnungsrede des Präsidenten der Deutschen Gesellschaft für Viszeralchirurgie (DGVC), Professor Reinhard Bittner, anlässlich der 123. Jahrestagung der Deutschen Gesellschaft für Chirurgie in Berlin.
}

Die aktuelle angespannte Lage im Gesundheitssystem konfrontiert auch die Chefärzte zunehmend mit ökonomischen und logistischen Problemen. Das kann aber nicht heißen, dass für die Ausübung unseres Berufs ein Master of Business Arts zwingende Voraussetzung ist, auch wenn Kenntnisse in diesem Bereich unabdingbar sind, um auch weiterhin akzeptierte Gesprächspartner für unsere Geschäftsführungen und Klinikleitungen zu bleiben. Stattdessen sollten wir uns in erster Linie auf den Erwerb und den Erhalt unserer fachlichen Kompetenz konzentrieren. Dabei dürfen uns aber die wirtschaftlichen Belange unseres Krankenhauses nicht gleichgültig sein! Dennoch ist es meine Überzeugung, dass gute Medizin letztlich auch im ökonomisierten Prozess unseres Gesundheitssystems kostengünstiger ist.

Was können wir tun? Was sind die Brennpunkte? Was wollen wir?

Ich bin überzeugt, angehende Chirurgen wollen nach wie vor in erster Linie operieren lernen. Die Problematik, mit der sich der junge Chirurg während seiner Ausbildung konfrontiert sieht, betrifft die Arbeitszeit, die Arbeitsbedingungen, die Entlohnung und die berufliche Perspektive.

Ein wesentlicher Punkt ist, dass sich die Arbeit ungeheuer verdichtet hat, nicht zuletzt auch durch den enormen Zuwachs an Bürokratie. Der Chirurg ist pausenlos im Einsatz, muss Überstunden machen und hat nicht selten trotzdem das Gefühl, die Arbeit nicht geschafft zu haben. Für Ausbildung, geschweige denn Kreativität, vor allem aber auch für Freude am Umgang mit dem Patienten und am Operieren verbleibt kaum Zeit. Das schafft Unzufriedenheit!

Wie sah es in früheren Zeiten aus? Kürzlich wurde mir von einem Patienten ein Band des «Archiv für klinische Chirurgie» (dem Vorläufer von Langenbeck's Archives for Surgery) aus dem Jahr 1876 geschenkt. In diesem Band findet sich die gesamte OP-Statistik des Jüdischen Krankenhauses in Berlin über 3 Jahre. In diesen 3 Jahren wurden im Jüdischen Kran-

\begin{tabular}{ll}
\hline KARGER & @ 2006 S. Karger GmbH, Freiburg \\
Fax +49 7614520714 & Accessible online at: \\
$\begin{array}{l}\text { E-mail Information@Karger.de } \\
\text { www.karger.com }\end{array}$ & www.karger.com/cga
\end{tabular}


kenhaus nicht mehr als 300 Operationen durchgeführt - eine Anzahl die eine heutige chirurgische Abteilung in weniger als einem Monat zu bewältigen hat! Während noch vor 20 Jahren ein Patient mit einer Sigmaresektion wegen eines Karzinoms zirka 4 Wochen im Krankenhaus zu verbleiben hatte, werden heute lediglich 5-7 Tage gefordert. Wenn heute ein Stationsarzt am Donnerstag Bereitschaftsdienst hat, Freitag früh nach Hause geht und am Montag wiederkommt, kann es sein, dass die Belegung seiner 18-Betten-Station komplett gewechselt hat. Die Liste der Beispiele ließe sich endlos fortsetzen. Kein Zweifel, die Arbeitsbelastung für den Einzelnen hat enorm zugenommen.

Eines scheint sicher: die Arbeit muss entzerrt und besser strukturiert werden, und sie muss befreit werden von überwucherndem bürokratischem Ballast. Die Organisation der Forschung ist ein Schwachpunkt des jetzigen Systems, der verheerende Folgen haben kann. Die Forschung muss professionalisiert werden, denn nur die wissenschaftlich basierte Chirurgie wird letztlich auch erfolgreich sein. Sie ist der Schlüssel zur Zukunft. Es kann nicht sein, dass Forschung überwiegend in der freien Zeit, nach Dienstschluss und an den Wochenenden, stattfinden muss. Sie muss integraler Bestandteil der Regelarbeitszeit sein. Nur so können wir wieder dahin kommen, wo wir einmal waren, als auch Amerikaner nach Deutschland pilgerten, um sich über den aktuellen Stand der Chirurgie zu informieren.

Noch kann Deutschland einiges bieten. In zahlreichen Kliniken wird hervorragende Arbeit geleistet, mit weltweit führenden Resultaten z.B. in der Pankreas- und laparoskopischen Chirurgie (hier mit den bisher weltweit größten publizierten Einzelserien z.B. in der minimal invasiven, der Gallenblasen-, Hernien- und Kolon- und Rektumchirurgie). Es lohnt sich, in Deutschland zu bleiben! Und so schlecht sind die Perspektiven auch nicht: es ist immer noch attraktiv, Spezialist und leitender Arzt zu werden - und diese Positionen besitzen in Deutschland bei weitem mehr Attraktivität als in den Ländern, die heute so eine große Anziehungskraft auf junge Ärzte ausüben.

Der Erfolg der Viszeralchirurgie - nicht zuletzt auch im Rahmen des betriebswirtschaftlichen Konzepts eines Krankenhauses - wird mehr und mehr bestimmt von der interdisziplinären Zusammenarbeit, und hier im Besonderen von der $\mathrm{Zu}-$ sammenarbeit mit der Gastroenterologie. Wohl abgestimmte Synergien in der Behandlung führen zu verbesserter Kosteneffektivität und tragen wesentlich zu einer gesunden finanziellen Basis des Krankenhauses bei. In dem von den DRGs dominierten Abrechnungssystem entscheidet das rasche und zielgerichtete Ineinandergreifen von Diagnostik und Therapie über Gewinn oder Verlust. Die innige Verzahnung von Viszeralchirurgie und Gastroenterologie bis hin zur Viszeralmedizin ist daher im Rahmen der Erfordernisse der neuen Strukturen unverzichtbar.

Unsere Zeitschrift ChIRURgische Gastroenterologie INTERDISZIPLINÄR hat diese notwendige Entwicklung bereits vor Jahren erkannt und in den überwiegend interdisziplinär strukturierten Themenheften gefördert und Ausdruck verliehen, wobei bewusst auf Originalarbeiten verzichtet wurde. Die Themenhefte verstehen sich als Fortbildung auf höchstem Niveau, sowohl für den Chirurgen als auch für den Gastroenterologen. Dieses unverwechselbare Charakteristikum unserer Zeitschrift soll in Zukunft durch CME-Artikel verstärkt werden, die von Professor Heidecke und Professor Lerch aus Greifswald herausgegeben werden.

Wenn das vorliegende Heft dem bewährten Konzept nicht folgt, ist dies eine Ausnahme und nicht beispielgebend für die zukünftige Entwicklung. Die Ausnahme ist darin begründet, dass eine Vielzahl von Kollegen aus Osteuropa, der Türkei und auch aus China auf unsere Zeitschrift aufmerksam geworden sind und uns eine Vielzahl von Originalarbeiten und Fallberichten zur Veröffentlichung zugeschickt haben. Wir haben uns entschlossen, eine Reihe dieser Arbeiten zu akzeptieren und gebündelt im vorliegenden Heft zu publizieren. Die Welt rückt näher zusammen, Globalisierung ist bereits mehr als nur ein Schlagwort. Die Chirurgische Gastroenterologie INTERDISZIPLINÄR kommt dieser Entwicklung nach und erlaubt ihren Lesern mit dem vorliegenden Heft einen Einblick in medizinische Prozesse in Ländern, die bislang aufgrund der politischen Konstellationen weitgehend von der internationalen Kommunikation ausgeschlossen waren. Wir hoffen sehr, dass Sie die Beiträge mit Interesse lesen und uns vielleicht auch die eine oder andere Rückmeldung geben.

Mit der nächsten Ausgabe wird dann das bewährte Konzept unserer Zeitschrift - möglicherweise bereits durch einen ersten CME-Artikel ergänzt - weitergeführt werden. 